\title{
Magnetic Field Reversal Resulting from a Dynamo Process in a Spherical Shell
}

\author{
Y. Honkura, T. IIJIma, and M. Matsushima \\ Department of Earth and Planetary Sciences, Tokyo Institute of Technology, Tokyo 152, Japan
}

(Received April 22, 1992; Revised August 11, 1992)

The magnetic field is one of the intrinsic properties of the Earth, originating in a magnetohydrodynamic (MHD) process in the liquid outer core. It also exhibits a variety of features of secular variation; most typically, reversals of the polarity of the axial dipole term and westward drifts of the non-zonal terms. Here we report on the results of our simulations of an MHD dynamo process in a rotating spherical shell and demonstrate that the above two typical features of the Earth's magnetic field can be understood quite naturally without any specific conditions. We also examine how the virtual geomagnetic pole (VGP) moves, in our MHD dynamo model, during transitional stages from one polarity to the other, and show that some features of the transitional behaviour derived from the paleomagnetic data can be seen in our dynamo model as well.

\section{Introduction}

The magnetic field is one important property of the Earth, and the mechanism for maintaining the Earth's magnetic field has been understood in terms of a magnetohydrodynamic (MHD) process in the Earth's core. Detailed observational studies of the Earth's magnetic field disclosed a variety of dynamical features of its secular variation. One typical example is the reversal of polarity of the axial dipole constituent, which has been considered to persist throughout the history of the Earth's magnetic field, known as the geocentric axial dipole hypothesis (MERILL and MCELHINNY, 1983). Another example is the westward drifts of the non-zonal constituents, with the drift velocity amounting to 0.2 0.3 degrees/year (e.g. YUKUTAKE and TACHINAKA, 1969), although detailed studies of secular variation (BLOXHAM and GuBBINS, 1985) indicate that such drifts are not necessarily an overall feature but are rather spatially confined to the Atlantic hemisphere $\left(90^{\circ} \mathrm{E}\right.$ to $\left.90^{\circ} \mathrm{W}\right)$ (GubBins and Bloxham, 1987).

The polarity reversal has been shown to arise spontaneously from non-linear interactions between the rotational velocity and the magnetic field in some coupled-disk dynamo models (e.g. SHIMIZU and HonkURA, 1985). Although the governing equations for these models may reflect, in an extremely simplified form, an essential aspect of a nonlinear dynamo process, they have been considered to be merely a remote analogue for the geodynamo. 


\section{MHD Dynamo Formulation}

A more realistic approach is certainly to solve the equations relevant to the geodynamo; they are the Navier-Stokes equation for the velocity field, the induction equation for the magnetic field, and the energy equation for a source which drives fluid motion. As to the force to drive a convective motion, two mechanisms have been proposed; the thermal and the compositional convections. Here we assume a thermal convection, because the thermal instability of fluid in a rotating spherical shell has been well known (CHANDRASEKHAR, 1961) and hence the reliability of our computations can be checked. Then the equations are given in non-dimensional forms as

$$
\begin{aligned}
& \nabla \cdot \boldsymbol{V}=0 \\
& \frac{1}{P_{r}} \frac{\partial}{\partial t}(\nabla \times \boldsymbol{V})+\frac{1}{P_{r}} \nabla \times[(\nabla \times \boldsymbol{V}) \times \boldsymbol{V}] \\
& =\nabla^{2}(\nabla \times \boldsymbol{V})+R_{a} \nabla \times(\Theta \boldsymbol{r})+T_{a}^{\frac{1}{2}} \nabla \times(\boldsymbol{V} \times \boldsymbol{\Omega})+\nabla \times[(\nabla \times \boldsymbol{B}) \times \boldsymbol{B}] \\
& \frac{\partial \Theta}{\partial t}+(\boldsymbol{V} \cdot \nabla) \Theta=-(\boldsymbol{V} \cdot \nabla) T_{0}+\nabla^{2} \Theta \\
& \nabla \cdot \boldsymbol{B}=0 \\
& \frac{\partial \boldsymbol{B}}{\partial t}=\frac{P_{r}}{P_{m}} \nabla^{2} \boldsymbol{B}+\nabla \times(\boldsymbol{V} \times \boldsymbol{B})
\end{aligned}
$$

where the thickness of the shell $(d)$ is taken as the characteristic length scale, the typical time for thermal diffusion $\left(d^{2} / \kappa\right)$ as the characteristic time, the temperature difference between the top and the bottom of the shell $(\Delta T)$ as the temperature, and $(\nu \kappa \rho \mu)^{1 / 2} / L$ as the magnetic field. In this magnetic field scaling, the diffusion term is comparable with the Lorentz force term in the Navier-Stokes equation. $\Theta$ is taken as the deviation from the static solution $T_{0}$, which is written as $T_{0}=C_{1}+C_{2} / r\left(C_{1}, C_{2}\right.$ are constants) for the condition of constant temperatures at the outer and inner boundaries without any thermal sources in the interior. Dimensionless numbers included in the above equations are Rayleigh $\left(R_{a}\right)$, Prandtl $\left(P_{r}\right)$, Taylor $\left(T_{a}\right)$, and magnetic Prandtl $\left(P_{m}\right)$ numbers. They are expressed as $R_{a}=g \alpha \Delta T d^{4} / \kappa \nu, P_{r}=\nu / \kappa, T_{a}=\left(2 \Omega d^{2} / \nu\right)^{2}$, and $P_{m}=\nu / \eta$.

The method of our numerical evaluation is as follows. We separate the velocity and the magnetic fields into the poloidal and the toroidal constituents as in most studies of fluid dynamics and dynamos in a spherical body; $\boldsymbol{V}=\nabla \times \nabla \times\left(V_{S} \boldsymbol{r}\right)+\nabla \times\left(V_{T} \boldsymbol{r}\right), \boldsymbol{B}=$ $\nabla \times \nabla \times\left(B_{S} \boldsymbol{r}\right)+\nabla \times\left(B_{T} \boldsymbol{r}\right)$. We then expand each variable into a series of spherical harmonics for $\theta$ and $\phi$, and trigonometric functions for $r$ as

$$
\begin{aligned}
V_{S} & =\sum_{l=1}^{L} \sum_{m=0}^{l} \sum_{n=1}^{N} V_{S l n}^{m}(t) Y_{l}^{m}(\theta, \phi) \sin \left(n \pi\left(r-r_{i}\right)\right) \\
V_{T} & =\sum_{l=1}^{L} \sum_{m=0}^{l} \sum_{n=1}^{N} V_{T l n}^{m}(t) Y_{l}^{m}(\theta, \phi) r \cos \left((n-1) \pi\left(r-r_{i}\right)\right) \\
\Theta & =\sum_{l=0}^{L} \sum_{m=0}^{l} \sum_{n=1}^{N} \Theta_{l n}^{m}(t) Y_{l}^{m}(\theta, \phi) \sin \left(n \pi\left(r-r_{i}\right)\right)
\end{aligned}
$$



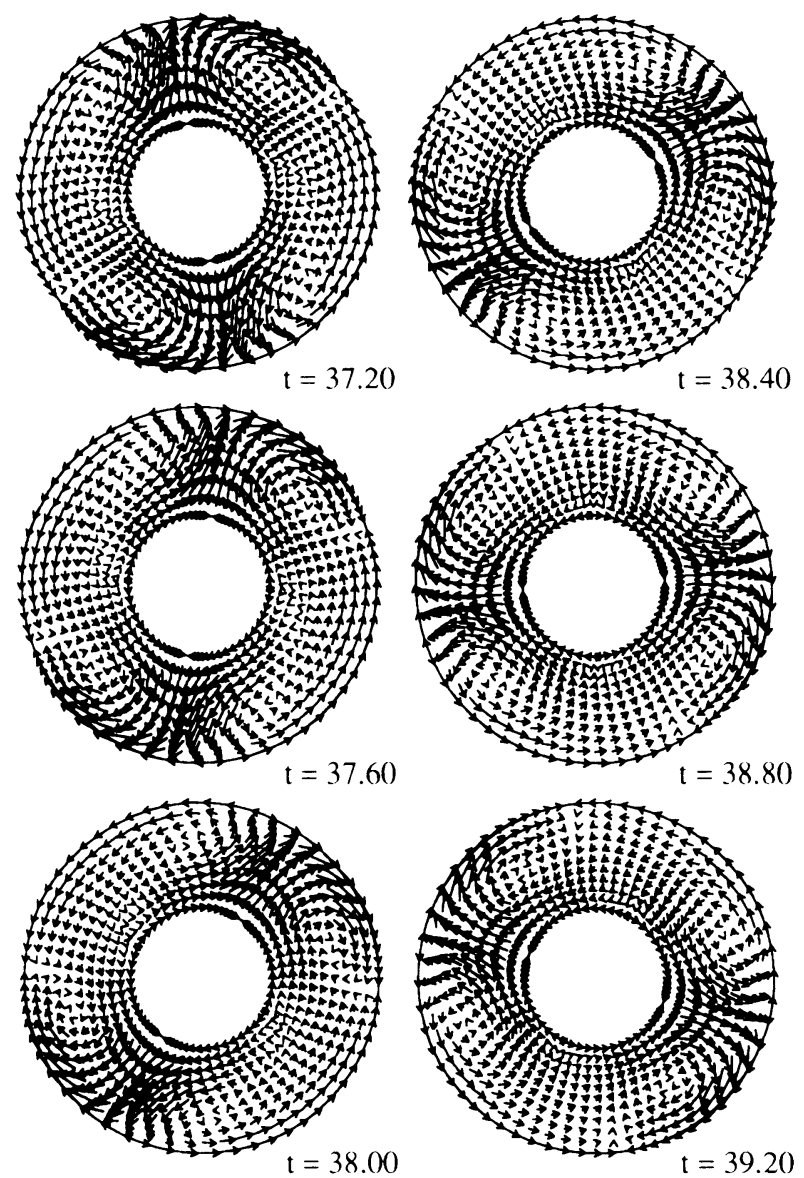

Fig. 1. Convective motion in the equatorial plane for dimensionless time $t=37.20 \sim 39.20$. Model parameters are as follows: $P_{r}=1, T_{a}=100, R_{a}=900, P_{m}=30$. The $P_{2}^{2}$ component is dominant and the pattern slowly drifts toward the west.

$$
\begin{aligned}
B_{S} & \left.=\sum_{l=1}^{L} \sum_{m=0}^{l} \sum_{n=1}^{N} B_{S l n}^{m}(t) Y_{l}^{m}(\theta, \phi) \cos \left(\alpha_{l n} r-\beta_{l n}\right)\right) \\
B_{T} & =\sum_{l=1}^{L} \sum_{m=0}^{l} \sum_{n=1}^{N} B_{T l n}^{m}(t) Y_{l}^{m}(\theta, \phi) \sin \left(n \pi\left(r-r_{i}\right)\right)
\end{aligned}
$$

where

$$
\begin{aligned}
\alpha_{l n} \tan \left(\alpha_{l n} r_{o}-\beta_{l n}\right)-\frac{l+1}{r_{o}} & =0 \\
\alpha_{l n} \tan \left(\alpha_{l n} r_{i}-\beta_{l n}\right)+\frac{l}{r_{i}} & =0
\end{aligned}
$$

and $r_{i}$ and $r_{o}$ are the radii of the inner and the outer boundaries, so that the boundary 

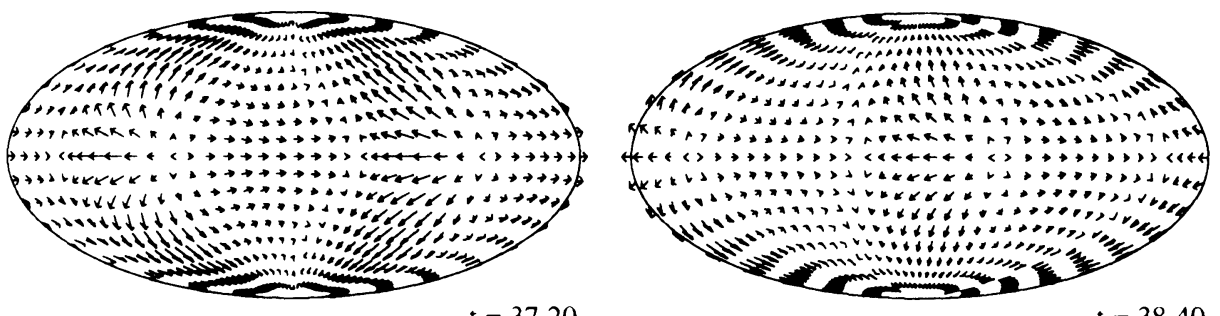

$$
\mathrm{t}=37.20
$$
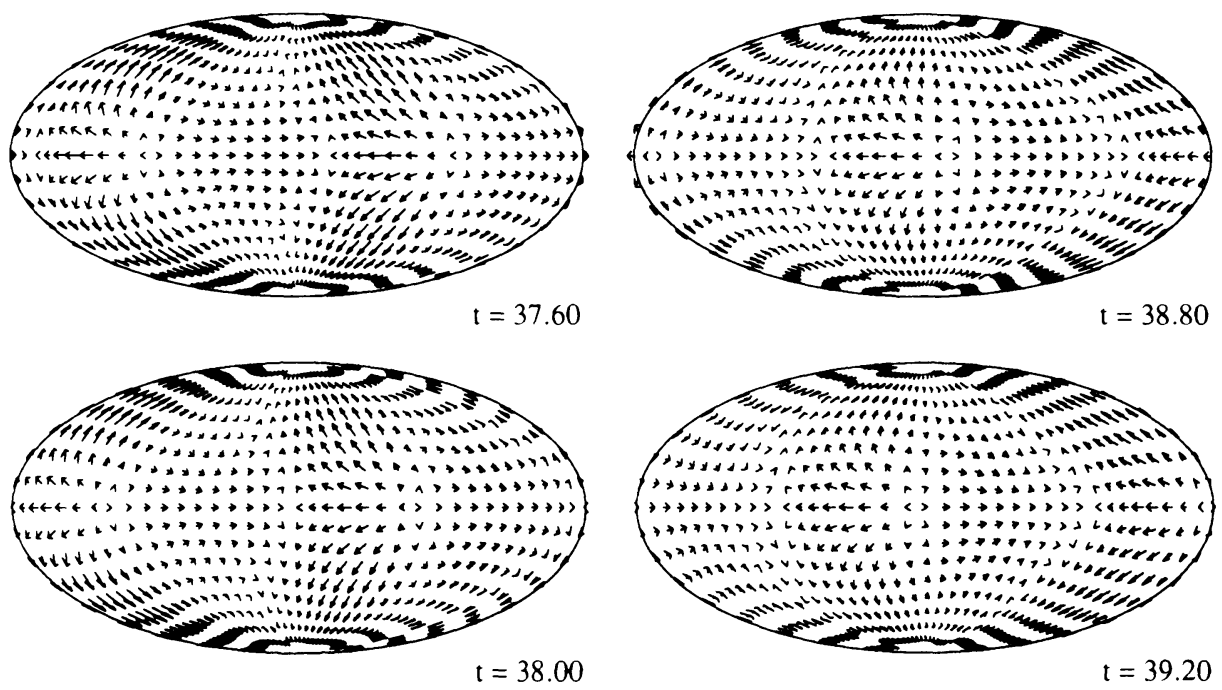

Fig. 2. Convective motion near the outer boundary for dimensionless time $t=37.20 \sim 39.20$.

conditions

$$
\begin{aligned}
V_{S l}^{m}(r) & =0 \\
\frac{\partial^{2}}{\partial r^{2}} V_{S l}^{m}(r) & =0 \\
\frac{\partial}{\partial r}\left(\frac{V_{T l}^{m}(r)}{r}\right) & =0 \\
\Theta_{l}^{m}(r) & =0 \\
\left(\frac{\partial}{\partial r}+\frac{l+1}{r}\right) B_{S l}^{m}(r) & =0 \quad \text { at } r=r_{o} \\
\left(\frac{\partial}{\partial r}-\frac{l}{r}\right) B_{S l}^{m}(r) & =0 \quad \text { at } r=r_{i} \\
B_{T l}^{m}(r) & =0
\end{aligned}
$$

are all satisfied. We then examine time evolution of $V_{S l n}^{m}(t), V_{T l n}^{m}(t), \Theta_{l n}^{m}(t), B_{S l n}^{m}(t)$, $B_{T l n}^{m}(t)$. We fixed the ratio of the inner to outer radii as 0.4 (nearly the same as the Earth's outer core), and assumed that the regions outside the shell are non-conducting. The inner core cannot be considered as non-conducting, but this assumption is unlikely to be very crucial because of its small size (ZHANG and BUSSE, 1990). We also assumed 
that the outer and inner boundaries are both free. We then examine time evolution of $V_{S l n}^{m}(t), V_{T l n}^{m}(t), \Theta_{l n}^{m}(t), B_{S l n}^{m}(t), B_{T l n}^{m}(t)$. We used the fourth-order Runge-Kutta scheme to avoid a possible numerical error at the expense of computation time.

\section{Polarity Reversals and Westward Drift}

Some of the physical quantities of the Earth's core have been estimated only poorly and hence dimensionless numbers are not well constrained. If we are to examine a realistic dynamo process relevant to the Earth's core, we must examine time evolution for a wide range of dimensionless numbers. Instead, in this report, we focus our attention on the possibility of polarity reversal of the magnetic field in a homogeneous MHD dynamo model. For this purpose we started computations with $R_{a}$ slightly larger than the critical value for the onset of a thermal convection for a slowly rotating spherical shell.
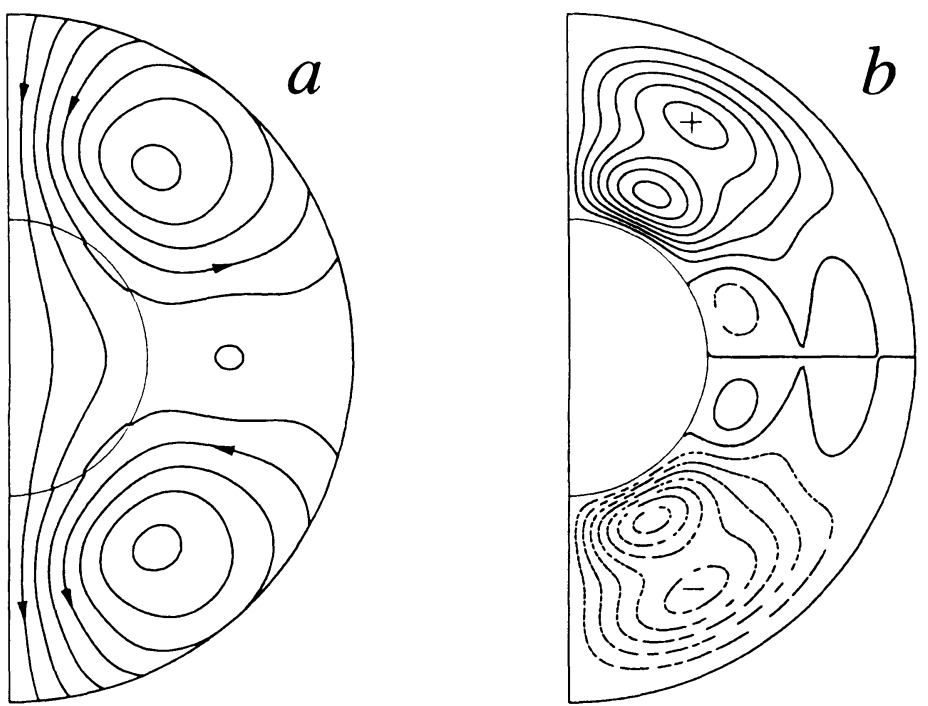

Fig. 3. Axisymmetric constituents of the poloidal and toroidal magnetic fields for $t=37.20:(a)$ magnetic lines of force for the poloidal component and $(b)$ contours for the toroidal component.

Here we report on the results for $P_{r}=1, T_{a}=100, R_{a}=900$. The truncation level is set at $L=N=4$. In this case, a westward drifting convection pattern characterized by $P_{2}^{2} \cos (2 \phi+\delta)$ prevails, as shown in Figs. 1 and 2 . If the velocity field is symmetric with respect to the equator, as in $P_{2}^{2}$ pattern, the magnetic field can be treated separately for the antisymmetric (EA) and the symmetric (ES) cases, respectively. They are often referred to as the dipole (EA) and the quadrupole (ES) families (MERrill and MCFAdDEn, 1988; HofFMAN, 1991). We report only on the dipole (EA) family. For small $P_{m}$ values an initially given magnetic field turned out to be subject to eventual decay with slight fluctuations during the decay. We then 


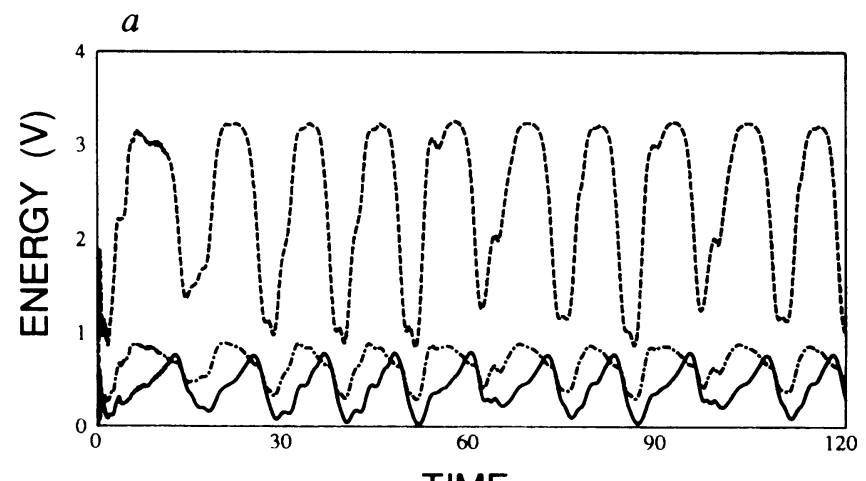

TIME

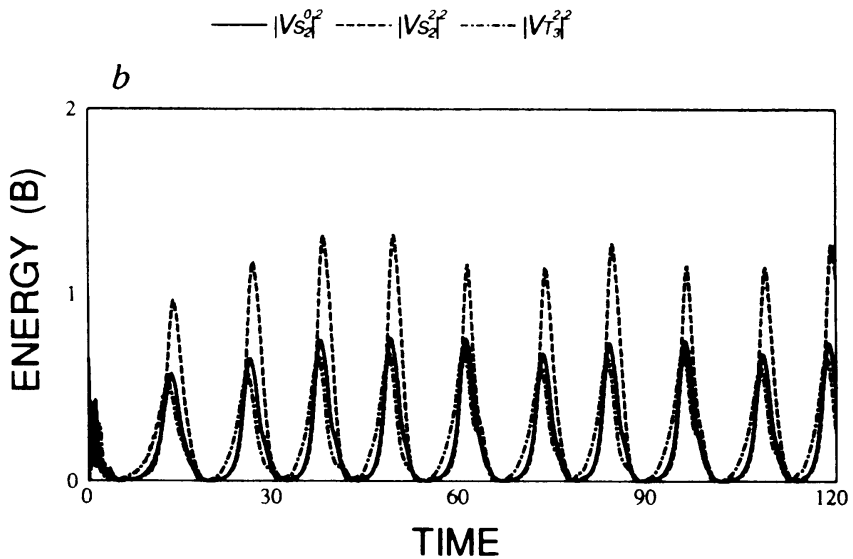

$-\left.B B_{1}^{0}\right|^{2}-\left.B_{s}^{0}\right|^{2}-\cdots \cdots\left|B S_{s}^{2}\right|^{2}$

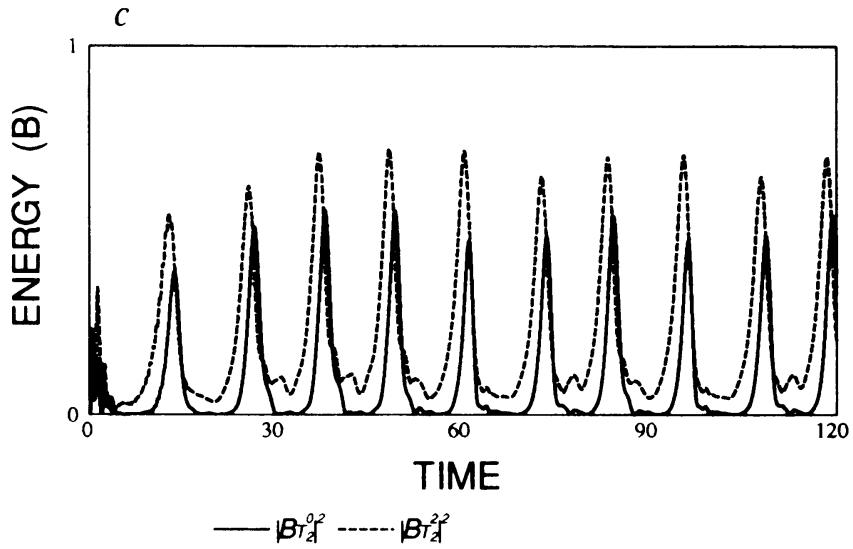

Fig. 4. (a) Evolution of the dominant velocity fields averaged for the entire shell with respect to the dimensionless time. (b) Evolution of the dominant poloidal magnetic fields averaged for the entire shell with respect to the dimensionless time. (c) Evolution of the dominant toroidal magnetic fields averaged for the entire shell with respect to the dimensionless time. 
increased $P_{m}$ gradually and found that the magnetic field can be sustained for $P_{m}=30$. Figure 3 shows the axisymmetric constituents of the poloidal and toroidal magnetic fields in the meridional plane.

Figure 4 shows time evolution of the velocity and the magnetic fields, for the above dimensionless numbers, in terms of their energy for the most dominant terms. As the velocity field grows, the magnetic field tends to grow rather rapidly. This behaviour is understood as resulting from the enhancement of the generation term in the induction equation. As the magnetic field grows, the velocity field is rapidly reduced because of the suppression due to the enhanced Lorentz force in the Navier-Stokes equation. Then the velocity field grows again. This process is repeated with fluctuations in maximum velocity and magnetic fields. It is interesting to note that such a behaviour has already been noted in the Bullard disk dynamo model (BULlaRD, 1955). In this sense, disk dynamo models may be considered to represent an essential aspect of a homogeneous MHD dynamo, at least for the above dimensionless numbers.

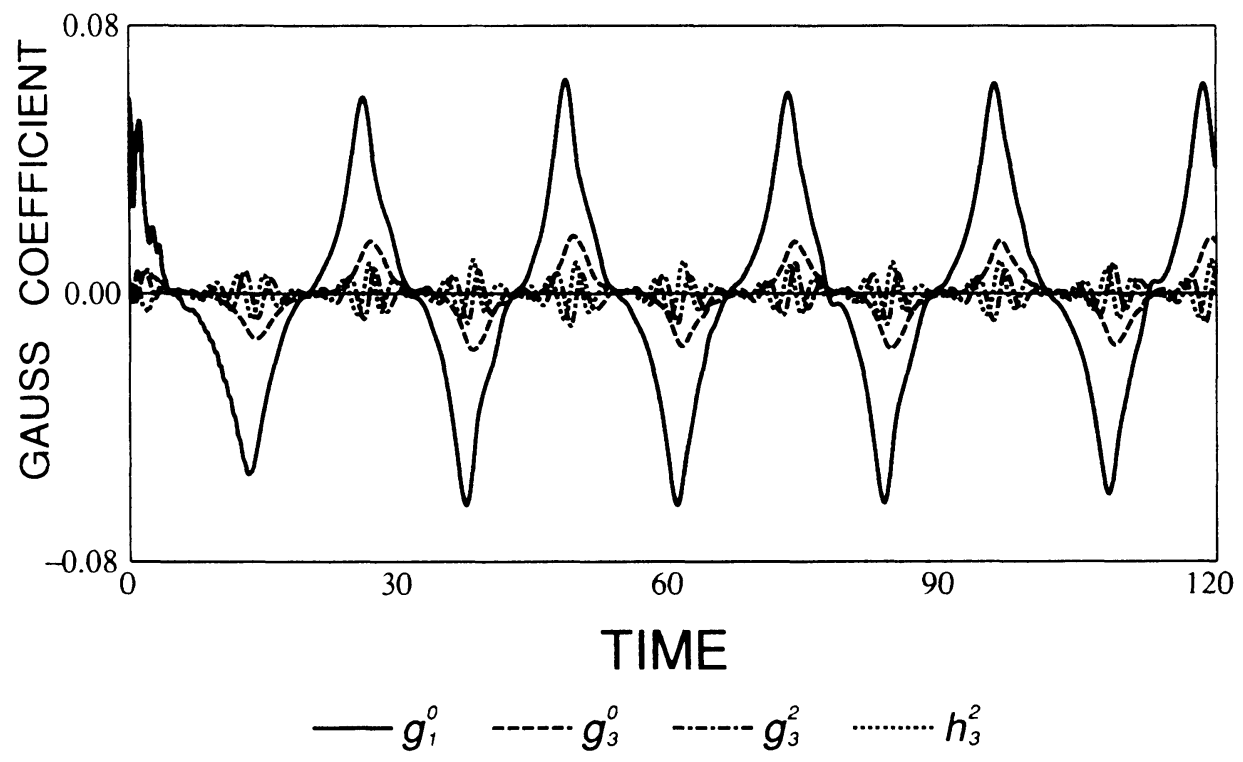

Fig. 5. Evolution of the dominant Gauss coefficients with respect to the dimensionless time. The $g_{1}^{0}$ and $g_{3}^{0}$ terms change rather periodically, whereas the $g_{3}^{2}$ and $h_{3}^{2}$ terms fluctuate more rapidly.

It is convenient to represent the poloidal magnetic field in terms of the Gauss coefficients so that secular variations can be compared with the existing geomagnetic data. For this purpose we added an insulating shell corresponding to the mantle of the Earth and evaluated the Gauss coefficients for such a model. We then found an exciting result; the $g_{1}^{0}$ (axial dipole) term reverses, rather periodically, its sign together with the $g_{3}^{0}$ term, as shown in Fig. 5. This result implies that it is possible for polarity reversals to occur spontaneously in a homogeneous MHD dynamo, although we cannot 
generalize this possibility with the present simple model. It is also interesting to note that the behaviour of the $g_{1}^{0}$ and $g_{3}^{0}$ terms is somehow similar to oscillatory polarity reversals which occasionally appear in a modified Bullard disk dynamo model, called the Cox model (Honkura and Matsushima, 1988).

It should be noted here that similar results have been derived by OLSON and HAGEe (1990) and HAGEe and Olson (1991), although their results are based on specific types of $\alpha$-effect and hence our results cannot be compared directly with their results.

Figure 6 shows a time-dependent feature of the radial component of the magnetic field at the outer boundary corresponding to the core-mantle boundary when the shell is considered as the Earth's outer core. In this particular case, the $P_{3}^{2}$ constituent is the most dominant non-zonal term. We see two negative foci in the northern hemisphere and corresponding positive foci in the southern hemisphere. As the time evolves, such a pattern moves to the west. Thus the present model also turns out to be capable of simulating the westward drift of the Earth's magnetic field.
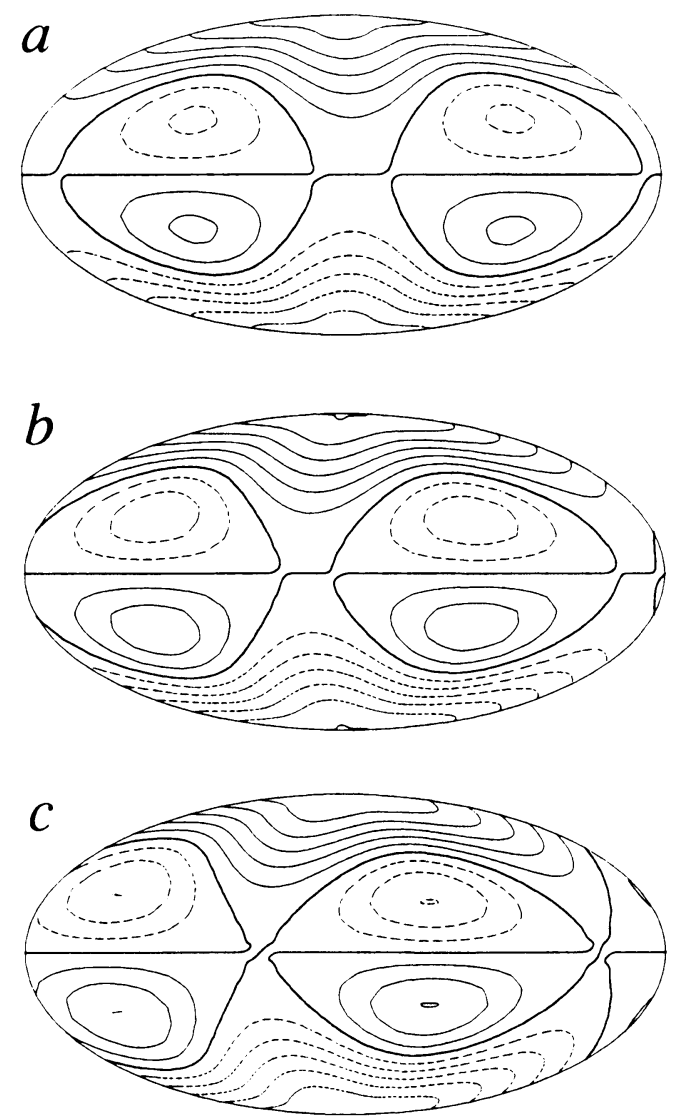

Fig. 6. Contours of the radial magnetic field at the outer boundary (corresponding to the coremantle boundary when the shell is regarded as the Earth's outer core) at $t=37.20(a), t=37.60$ $(b), t=38.00(c)$. The pattern drifts toward the west. 

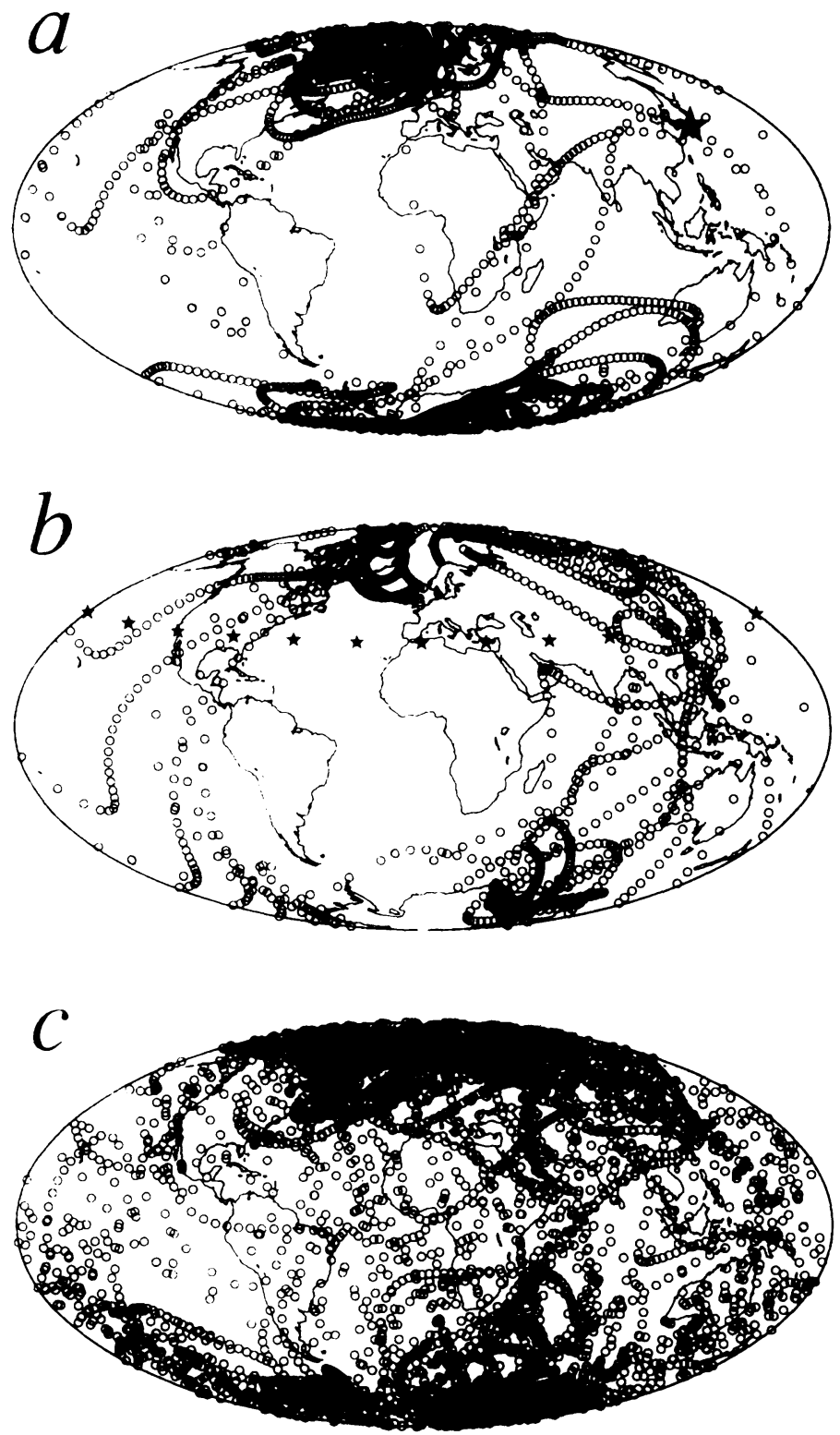

Fig. 7. (a) VGP trajectories on a spherical surface (corresponding to the Earth's surface) derived from the magnetic field at a site, denoted by a star symbol, located at $35^{\circ} \mathrm{N}, 135^{\circ} \mathrm{E}$. One of the trajectories during transitions passes near the observation site. Excursion-like trajectories are also seen. (b) VGP trajectories for one particular transitional stage derived from the magnetic fields at sites, denoted by star symbols, at $0^{\circ}, 30^{\circ} \mathrm{E} \& \mathrm{~W}, 60^{\circ} \mathrm{E} \& \mathrm{~W}, 90^{\circ} \mathrm{E} \& \mathrm{~W}, 120^{\circ} \mathrm{E} \& \mathrm{~W}, 150^{\circ} \mathrm{E}$ $\& \mathrm{~W}, 180^{\circ}$ along the latitude of $35^{\circ} \mathrm{N}$. A tendency of clustering near a meridional great circle seems to be recognized. (c) VGP trajectories for all the transitional stages derived for the same sites along the latitude of $35^{\circ} \mathrm{N}$. No clear clustering can be seen any longer. 


\section{Discussion}

One may argue against the present results from the standpoint of a realistic geodynamo, since some dimensionless numbers are far below likely ranges for the Earth's outer core and hence much stress should not be placed on the relevance to the geodynamo. We admit such a claim and progress should be made toward a more realistic dynamo model.

A more severe problem would be a possible insufficient truncation level. In our preliminary results, the truncation level seems to affect the magnetic field behaviour, suggesting that general MHD dynamos are very unstable. This problem may be avoided if we always select parameter values which are slightly larger than the critical values for the onset of instability of the magnetic field, again at the expense of the remoteness from a realistic geodynamo model. In any case, we need extensive numerical calculations to solve this important yet extremely difficult problem.

What we point out here is only the possibility that the polarity reversal and the westward drift can occur spontaneously and we may not necessarily postulate any special mechanisms, for instance, at the outer (core-mantle) boundary.

It would be interesting to examine the virtual geomagnetic pole (VGP) path, since the geometry of VGP path during geomagnetic reversals has recently attracted much attention in paleomagnetism (TRIC et al., 1991; Clement and Kent, 1991; LAJ et al., 1991). One severe defect in the claim that there exists a preferential VGP path is the insufficient number of sampling of the paleofield and a resulting possible bias. In order to examine such a possibility, we determined a series of VGP positions for different locations along the latitude of $35^{\circ} \mathrm{N}$.

Figure 7 shows some examples. Locations of the continents are not meaningful, since there is no specific $\phi$-dependence in our model. Nonetheless we added them in the figure for comparison with the observations, as described below. There exists a near-sided VGP path (FULLER et al., 1979) which is similar to the observation at the Boso Peninsula, Japan, during the Matuyama-Brunhes transition (Nirssuma, 1971) (Fig. 7a). Occasionally an excursion-like VGP path swings toward the equator or even beyond the equator and soon returns near the pole. An apparent preferential path can be found for some transitions (Fig. 7b), but as the number of sampling increases, such a preferential path disappears (Fig. 7c). This implies that arguments about the existence of a preferential VGP path should be made carefully, although the correlation with the inhomogeneity of the lower mantle structure (LAJ et al., 1991) would suggest the existence of a preferential VGP path which may not otherwise be confirmed from insufficient paleomagnetic evidence.

We thank Peter Olson for his valuable comments. We originally submitted this paper as a letter with the limit of six printed pages, but we modified the manuscript so as to follow the comments and added some figures, resulting in a full paper. We are thankful to Masaru Kono, the editor responsible for this paper, for his suggestion of this transfer from a letter to a full paper. We also thank an anonymous referee for comments on the truncation level. This work was supported by the Yamada Science Foundation and also by the Ministry of Education, Science and Culture under the grant-in aid for scientific research on priority areas No. 02246105 and No. 03232105. Paper number 4 of the Deapartment of Earth and Planetary Sciences, Tokyo Institute of Technology. 


\section{REFERENCES}

Bloxham, J. and D. Gubbins, The secular variation of Earth's magnetic field, Nature, 317, 777-781, 1985.

Bullard, E. C.. The stability of a homopolar dynamo, Proc. Camb. Phil. Soc., 51, 744-760, 1955.

Chandrasekhar, S., Hydrodynamic and Hydromagnetic Stability, Oxford University Press, 1961.

Clement, B. M. and D. V. Kent, A southern hemisphere record of the Matuyama-Brunhes polarity reversal, Geophys. Res. Lett., 18, 81-84, 1991.

Fuller, M., I. Williams, and K. A. Hoffman, Paleomagnetic records of geomagnetic field reversals and the morphology of the transitional fields, Rev. Geophys. Space Phys., 17, 179-203, 1979.

Gubbins, D. and J. Bloxham, Morphology of the geomagnetic field and implications for the geodynamo, Nature, 325, 509-511, 1987.

Hagee, V. L. and P. Olson, Dynamo models with permanent dipole fields and secular variation, $J$. Geophys. Res., 96, 11673-11687, 1991.

Hoffman, K. A.. Long-lived transitional states of the geomagnetic field and the geodynamo families, Nature, 354, 273-277, 1991.

Honkura, Y. and M. Matsushima. Fluctuation of the nondipole magnetic field and its implication for the process of geomagnetic polarity reversal in the Cox model, J. Geophys. Res., 93, 11,631$11,642,1988$.

Laj, C., A. Mazaud, and R. Weeks, Geomagnetic reversal paths, Nature, 351, 447, 1991.

Merrill, R. T. and M. W. McElhinny, The Earth's Magnetic Field (Its History, Origin and Planetary Perspective), Academic Press, 1983.

Merrill, R. T. and P. L. MCFAdDEn, Secular variation and the origin of geomagnetic field reversals, J. Geophys. Res., 93, 11,589-11,597, 1988.

Nitssuma, N., Detailed study of the sediments recording of magnetic polarity transitions recorded in a high deposition rate deep-sea core, Tohoku Univ. Sci. Rep. 2nd Ser. (Geology), 43, 1-39, 1971.

Olson, P. and V. L. Hagee, Geomagnetic polarity reversals, transition field structure, and convection in the outer core, J. Geophys. Res., 95, 4609-4620, 1990.

Shimizu. M. and Y. HonkURA, Statistical nature of polarity reversals of the magnetic field in coupleddisk dynamo models, J. Geomag. Geoelectr., 37, 455-497, 1985.

Tric, E., C. Laj, C. Jéhanno, J.-P. Valet, C. Kissel, A. Mazaud, and S. Iaccarino, Highresolution record of the Upper Olduvai transition from Po Valley (Italy) sediments: support for dipolar transition geometry?, Phys. Earth Planet. Inter., 65, 319-336, 1991.

Yukutake, T. and H. Tachinaka, Separation of the earth's magnetic field into the drifting and standing parts, Bull. Earthq. Res. Inst. Univ. Tokyo, 47, 65-97, 1969.

ZHANG, K.-K. and F. H. Busse, Generation of magnetic fields by convection in a rotating spherical fluid shell of infinite Prandtl number, Phys. Earth Planet. Inter., 59, 208-222, 1990. 